\title{
Spontaneous antiferromagnetic order and strain effect on electronic properties of $\alpha$-graphyne
}

\author{
Baojuan Dong, ${ }^{1}$ Huaihong Guo, ${ }^{2,3}$ Zhiyong Liu, ${ }^{1}$ Teng Yang, ${ }^{1,3, *}$ \\ Peng Tao, ${ }^{4}$ Sufang Tang, ${ }^{1}$ Riichiro Saito, ${ }^{3}$ and Zhidong Zhang ${ }^{1}$ \\ ${ }^{1}$ Shenyang National Laboratory for Materials Science, \\ Institute of Metal Research, Chinese Academy of Sciences, \\ University of Chinese Academy of Sciences, Shenyang 110016, China \\ ${ }^{2}$ College of Sciences, Liaoning Shihua University, Fushun, 113001, China \\ ${ }^{3}$ Department of Physics, Tohoku University, Sendai 980-8578, Japan \\ ${ }^{4}$ Quanzhou Institute of Equipment Manufacturing, Haixi Institutes, \\ Chinese Academy of Sciences, Jinjiang, 362200, China
}

(Dated: July 30, 2021)

\begin{abstract}
Using hybrid exchange-correlation functional in $a b$ initio density functional theory calculations, we study magnetic properties and strain effect on the electronic properties of $\alpha$-graphyne monolayer. We find that a spontaneous antiferromagnetic (AF) ordering occurs with energy band gap $(\sim 0.5$ $\mathrm{eV})$ in the equilibrated $\alpha$-graphyne. Bi-axial tensile strain enhances the stability of AF state as well as the staggered spin moment and value of the energy gap. The antiferromagnetic semiconductor phase is quite robust against moderate carrier filling with threshold carrier density up to $1.7 \times 10^{14}$ electrons $/ \mathrm{cm}^{2}$ to destabilize the phase. The spontaneous AF ordering and strain effect in $\alpha$-graphyne can be well described by the framework of the Hubbard model. Our study shows that it is essential to consider the electronic correlation effect properly in $\alpha$-graphyne and may pave an avenue for exploring magnetic ordering in other carbon allotropes with mixed hybridization of $\mathrm{s}$ and $\mathrm{p}$ orbitals.
\end{abstract}

PACS numbers: 61.48.De, 68.55.ap, 62.25.-g, 61.46.-w, 81.05.ub

\section{INTRODUCTION}

Antiferromagnetic (AF) ordering in graphene induced by strain has recently attracted tremendous attention [1-6] and may supply a platform for both fundamental study of the Coulomb interaction and possible applications on switchable magnetic devices. However, the critical strain theoretically predicted[3] for a phase transition of graphene from semimetal to AF semiconductor is around $8 \%$, which is practically not easy to achieve in experiment and to demonstrate for potential applications. Graphyne as one type of graphene allotrope may serve as an alternative to realize such transition at much lower strain.

Graphyne has been proposed several decades ago[7]. Different from graphene with pure $\mathrm{sp}^{2}$ hybridization, graphyne has both sp hybridization in the linear C-C bond and $\mathrm{sp}^{2}$ hybridization at the hexagonal corner (see Fig. 1(a)). Thus the variety in bonding states renders graphyne an appealing material for studying the richness of electronic properties and tunability by strain. Recently, graphyne starts to attract renewed and increasing attention, mainly due to some direction-dependent properties of massless Dirac fermions and pseudospin state predicted in the graphyne systems[8-10]. Worth pointing out that most of the study on graphyne were purely based on the single-particle picture without many-body effect taken into account. However, single-particle picture has been proved insufficient, i.e., for the understanding of the Coulomb interaction in graphene[11] includ- ing the strain-induced AF state[5], and especially in graphyne with more localized sp states than the $\mathrm{sp}^{2}$ states in graphene. We will show that a strong spin ordering occurs in graphyne in contrast to graphene and that the Coulomb interaction in the structure with the sp C-C bond can be controlled by much smaller strain than that for graphene, like polyacetylene[12, 13], which may lead to a smaller critical strain or even zero strain for triggering spin-ordered state in graphyne.

To explore the phenomenon aforementioned, we studied electronic properties and strain effect in $\alpha$-graphyne monolayer by first-principles calculations, which are further interpreted by the Hubbard model. $\alpha$-graphyne has a similar hexagonal structure to graphene, but two extra carbon atoms are linearly inserted in $\alpha$-graphyne between two nearest neighbor carbon atoms in graphene (see Fig. 1) . Here we selected $\alpha$-graphyne[7] in terms of the following considerations: (1) $\alpha$-graphyne among all the proposed graphynes has the simplest structure with eight atoms per unit cell, but it still remains the main character of the graphyne family that both $\mathrm{sp}^{2}$ and sp hybridization coexist; (2) it has the same point symmetry group $\left(\mathrm{D}_{6 h}\right)$ as graphene, making a convenient analogy to graphene; (3) a model for estimating the effective hopping integral $\tilde{t}$ in $\alpha$-graphyne has been proposed [10], and can facilitate an evaluation of the critical value of $\mathrm{U} / \tilde{t}$ for a possible phase transition. In this model, the so-called effective hopping integral $\tilde{t}$ between two carbon atoms at the nearest hexagonal corner site in $\alpha$-graphyne can be described as $-t_{1}^{2} / t_{2}$ according to Kim et al.[10], where $t_{1}$ and $t_{2}\left(t_{1}, t_{2}>0\right.$ as shown in Fig. 1) are the hopping 


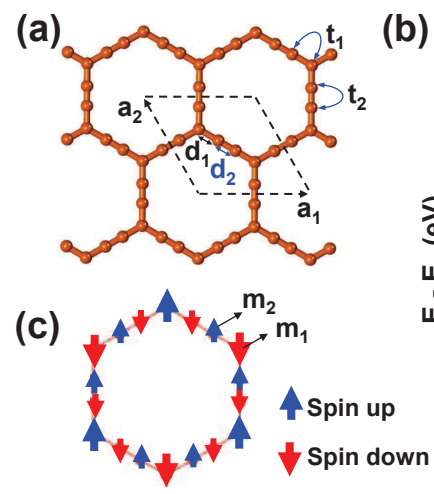

(b)

FIG. 1. (Color online) Spontaneous antiferromagnetic (AF) ordering of $\alpha$-graphyne monolayer in the electronic groundstate. (a) Atomic structure. (b) Energy band structure, bands for spin up and down are degenerate. (c) Spin texture of $\alpha$-graphyne.

integral values for the single $\mathrm{sp}^{2}$ bond and sp bond, respectively. Since $t_{1}$ is due to $\mathrm{sp}^{2}$ bond like C-C bond in graphene, $t_{1}$ can be approximately equal to that in graphene. $t_{2}$ is due to sp bond which is shorter than $\mathrm{sp}^{2}$ bond, and $t_{2}$ is larger than $t_{1}[14]$. Therefore, one expects that an effective $\tilde{t}$ in $\alpha$-graphyne is smaller than the hopping integral value in graphene. Such a small $\tilde{t}$ may be advantageous for a semimetal-antiferromagnetic semiconductor transition in $\alpha$-graphyne occurring under a reduced strain, since the kinetic exchange [15] is generally expressed by kinetic exchange interaction, $\tilde{t}^{2} / \mathrm{U}$, in which $\mathrm{U}$ is the on-site Coulomb interactions.

To evaluate the electron-electron interaction in $\alpha$ graphyne, we used hybrid exchange-correlation functional [16] in ab initio density functional theory calculations. We found that the critical strain $(\sim-3 \%$, compressive) for a semimetal-AF semiconductor transition in $\alpha$-graphyne is much reduced than that $(\sim+8 \%$, tensile $)$ of graphene. And more importantly, spontaneous AF spin ordering of semiconducting electronic ground state appears even at zero strain. Bi-axial tensile strain can enhance the stability of the AF state as well as the staggered spin moment and energy band gap at the zone-corner $\mathrm{K}$ point in the hexagonal Brillouin zone. The antiferromagnetic semiconductor phase is destabilized on carrier filling with a threshold carrier doping density up to $1.7 \times 10^{14}$ electrons $/ \mathrm{cm}^{2}$. A much smaller effective hopping integral in $\alpha$-graphyne than in graphene is responsible for the spontaneous AF ordering as is understood by the Hubbard model. The strain-enhanced stability of AF ground state in $\alpha$-graphyne may be observed experimentally even at finite temperature.

\section{COMPUTATIONAL TECHNIQUES}

In order to obtain the ground state and strain-induced properties in $\alpha$-graphyne, we used $a b$ initio density functional theory as implemented in the VASP code[17]. We use a periodic boundary condition with monolayer structures represented by a periodic array of slabs separated by a vacuum region $(\gtrsim 19.5 \AA)$. We use the projector augmented wave (PAW) pseudopotentials [18] and the Perdew-Burke-Ernzerhof (PBE) [19] exchangecorrelation functional. The Brillouin zone of the primitive unit cell of the $2 \mathrm{D}$ structures is sampled by $7 \times 7 \times 1 \mathrm{k}$ points [20]. We adopt $500 \mathrm{eV}$ as the electronic kinetic energy cutoff for the plane-wave basis and $10^{-6} \mathrm{eV}$ for a total energy difference between subsequent self-consistency iterations as the criterion for reaching self-consistency. All geometries are optimized using the conjugate gradient method [21], until none of the residual HellmannFeynman forces exceeds $2 \times 10^{-2} \mathrm{eV} / \AA$. Uniform strain $\varepsilon$ used in this study is defined as $\left(a-a_{0}\right) / a_{0}$, in which $a_{0}$ and $a$ are, respectively, the lattice constants without and with a strain.

To remedy the self-interaction error of the GGA calculations in carbon allotropes, we further performed the hybrid-functional (HSE06) calculations [16] to determine more accurately the ground state and strain effect. In the calculations, the PBE exchange energy and HartreeFock exchange energy were hybridized, along with the full PBE correlation energy.

The Hubbard model with Hartree-Fock mean-field approximation is also adopted here to get an essence of the emergent spontaneous AF order in $\alpha$-graphyne system.

$$
\begin{aligned}
\hat{H}_{H F} & =-\sum_{i j \sigma} \sum_{<p, q>} t_{p, q}^{i, j} \hat{c}_{i p \sigma}^{+} \hat{c}_{j q \sigma} \\
& +U \sum_{i p \sigma}\left[n_{i p \bar{\sigma}} \hat{c}_{i p \sigma}^{+} \hat{c}_{i p \sigma}-\frac{1}{2} n_{i p \bar{\sigma}} n_{i p \sigma}\right]
\end{aligned}
$$

in which $t_{p, q}^{i, j}$ represents hopping integral between the $\mathrm{p}^{t h}$ atom of the $\mathrm{i}^{\text {th }}$ unit cell and the $\mathrm{q}^{\text {th }}$ atom of the $\mathrm{j}^{\text {th }}$ unit cell, $\mathrm{U}$ the Coulomb repulsion, $\mathrm{i}, \mathrm{j}$ the index of lattice vectors, $\sigma$ spin index, p, q the index of atoms in the unit cell. Since the system is homogeneous, we can assume that number of particle at each atomic site has translational symmetry, $\mathrm{n}_{i p \sigma} \equiv \mathrm{n}_{p \sigma}$. Then, $\mathrm{n}_{p \sigma}$ is expressed by

$$
\begin{aligned}
n_{p \sigma} & =\left\langle\hat{c}_{i p \sigma}^{+} \hat{c}_{i p \sigma}\right\rangle \\
& =\frac{1}{N} \sum_{i}\left\langle\hat{c}_{i p \sigma}^{+} \hat{c}_{i p \sigma}\right\rangle \\
& =\frac{1}{N} \sum_{k}\left\langle\hat{c}_{k p \sigma}^{+} \hat{c}_{k p \sigma}\right\rangle,
\end{aligned}
$$

here $N$ is the number of $\mathrm{k}$ points in the $1^{\text {st }}$ Brillouine 
(a)

(c)
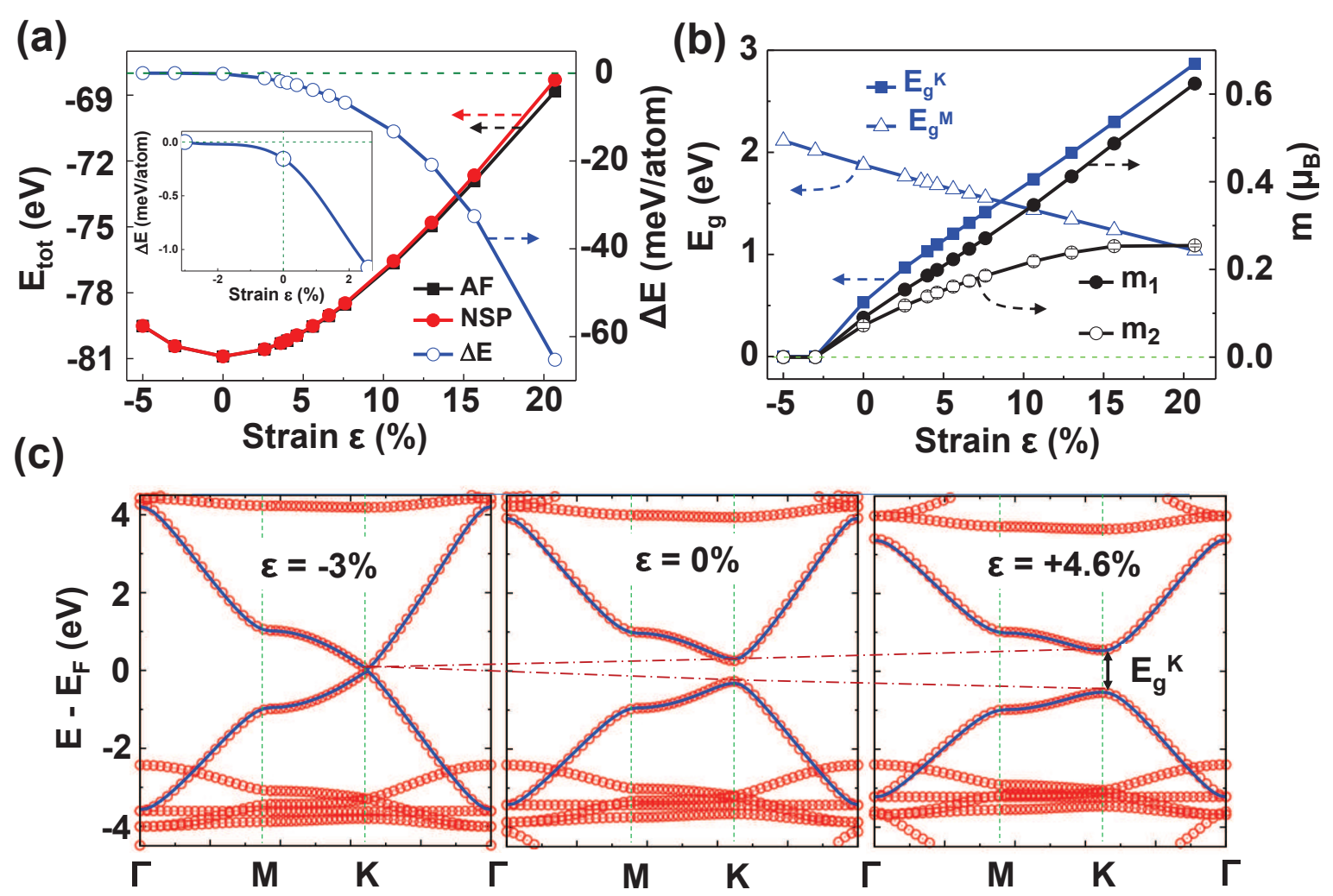

FIG. 2. (Color online) Strain effect on $\alpha$-graphyne. (a) The stability of antiferromagnetic ordering state (AF) with respect to non-polarized state (NSP) as a function of strain $\varepsilon$. The energy for the AF and NSP states are showed in black solid square and blue solid circle, respectively. And the energy difference $\Delta \mathrm{E}\left(=\mathrm{E}_{t o t}(\mathrm{AF})-\mathrm{E}_{\text {tot }}(\mathrm{NSP})\right)$ is showed in blue empty circle. (b) Energy band gap $\mathrm{E}_{g}^{M}$ and $\mathrm{E}_{g}^{K}$, and staggered spin moment $m_{1}$ and $m_{2}$ at two inequivalent atomic sites as a function of $\varepsilon$. (c) Band structures at three strains $\varepsilon=-3 \%, 0 \%$ and $+4.6 \%$. The empty circles represent the band structure from DFT calculations, and the blue solid lines are from the Hubbard model calculations which is to fit the DFT results. The brown dashed lines are used to highlight the band gap $\mathrm{E}_{g}^{K}$ evolving with strain.

zone, and $\hat{c}_{k p \sigma}^{+}\left(\hat{c}_{k p \sigma}\right)$ is the Fourier transform of $\hat{c}_{i p \sigma}^{+}$ $\left(\hat{c}_{i p \sigma}\right)$.

\section{RESULTS AND DISCUSSION}

Figure 1 shows the calculated results of $\alpha$-graphyne at zero strain $(\varepsilon=0)$. The optimized structure in Fig. 1(a) shows two different bonding length defined as $d_{1}=1.39$ $\AA$ and $d_{2}=1.22 \AA$ for the two distinct hybridizations $\mathrm{sp}^{2}$ and sp, respectively. In Fig. 1(b) we show the calculated electronic energy band. As shown in Fig. 1(b), $\alpha$-graphyne is a semiconductor with a direct energy band gap of around $0.5 \mathrm{eV}$ occurring at the zone corner $\mathrm{K}$ point of Brillouin zone. AF ordering appears with the degenerate electronic energy bands for spin up and down over the Brillouin zone. The inversion symmetry of the unit cell is broken by such an antiferromagnetic (AF) ordering, as shown in Fig. 1(c). The magnetic moment $m$ for the corner carbon $\left(m_{1}\right)$ is slightly larger than that of the edge carbons $\left(m_{2}\right)$, as shown in Fig. 2(b). The calculated results show that we have obtained both $\mathrm{AF}$ and semiconducting state in the $\alpha$-graphyne for $\varepsilon=0$ when taking the electronic correlation into account. While for graphene at zero strain there is no magnetic order, except for the boundary atoms at the zigzag edges [22-24].

To understand the spontaneous AF ordering, we applied uniform strain to $\alpha$-graphyne as a perturbation and calculated the strain effect on the electronic properties in $\alpha$-graphyne. In Fig. 2(a) we show the calculated total energies $\mathrm{E}_{\mathrm{tot}}$ of both non-spin-polarized (NSP) and AF states and also the energy difference $\Delta \mathrm{E}\left(=\mathrm{E}_{\mathrm{tot}}(\mathrm{AF})\right.$ $\left.\mathrm{E}_{\text {tot }}(\mathrm{NSP})\right)$ as a function of strain $\varepsilon$. Total energy minimum at zero strain indicates that the atomic structure is well optimized. From the inset of Fig. 2(a), we can see that $\Delta \mathrm{E}$ at zero strain is not zero but around 0.2 $\mathrm{meV} /$ atom, indicating that NSP state is less stable than the AF one. The energy difference becomes more pronounced with the increasing $\varepsilon$ and obviously tensile strain $(\varepsilon>0)$ can enhance the relative stability of AF to the NSP state, which may be understood from the increased Peierls distortion between $\mathrm{sp}$ and $\mathrm{sp}^{2}$ bond lengths due to tensile strain, as seen from $d_{1}$ and $d_{2}$ changing with $\varepsilon$ in Fig. 3(a). In contrast to the effect due to a tensile 

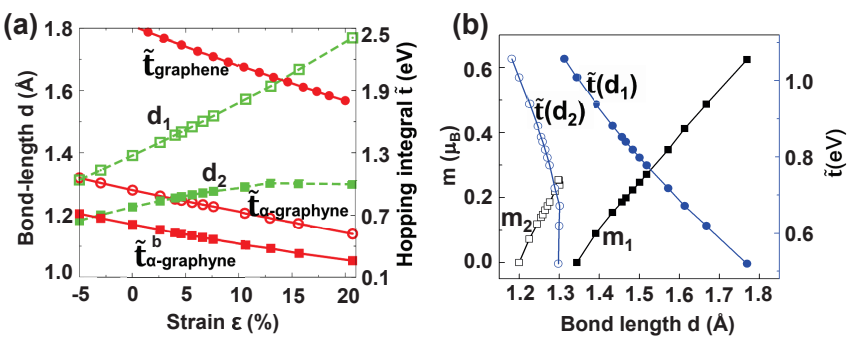

FIG. 3. (Color online) (a) Strain-dependent bond lengths $\left(\mathrm{d}_{1}\right.$ and $\mathrm{d}_{2}$ defined in Fig. 1(a)) and effective hopping integral $(\tilde{t})$ in $\alpha$-graphyne. Data of graphene in filled circles in (a) is from Lee et al.[3] and used for a compare, $\tilde{t}_{\alpha-\text { graphyne }}$ and $\tilde{t}_{\alpha-\text { graphyne }}^{b}$ are derived from two ways (refer to the main text for details). $\tilde{t}_{\alpha-\text { graphyne }}$ from half of $\mathrm{E}_{g}^{M}$ is used for more discussions. (b) $\tilde{t}$ and staggered magnetic moment as a function of bond lengths.

strain, a compressive strain $(\varepsilon<0)$ is found to reduce $\Delta \mathrm{E}$ till zero at $\varepsilon \sim-3.0 \%$.

Strain changes not only the relative stability of spinpolarized state, but also the magnitude of the band gap $\mathrm{E}_{g}$ and spin moment $m_{1}$ and $m_{2}$ in the semiconducting $\mathrm{AF}$ state. Figure 2(b) shows the strain dependence of $\mathrm{E}_{g}$ (blue solid square and open triangle) at the $\mathrm{K}$ and $\mathrm{M}$ points, respectively, and $m_{1}$ and $m_{2}$ (black solid and open circles). The $\mathrm{E}_{g}^{K}$ at the $\mathrm{K}$ point is a fundamental band gap of $0.5 \mathrm{eV}$ at $\varepsilon=0$. A compressive strain at $\varepsilon \sim$ $-3 \%$ closes the band gap, while a tensile strain increases $\mathrm{E}_{g}^{K}$ almost linearly. This trend is also shown in the strain-dependent electronic band structures in Fig. 2(c). Two dashed lines are used to highlight the band gap $\mathrm{E}_{g}^{K}$ changing with strain. On the other hand, $\mathrm{E}_{g}^{M}$ decreases with increasing tensile strain, which may have something to do with the electronic hopping which decreases with bond elongation due to tensile strain, as discussed more in Fig. 3. The spin magnetization has a similar trend as $\mathrm{E}_{g}^{K}$. At zero strain, $m_{1}$ and $m_{2}$ both have finite value $(\sim$ $\left.0.1 \mu_{B}\right)$ and go to zero at $\varepsilon \sim-3.0 \%$ where $\mathrm{E}_{g}^{K}$ drops to zero. Both $m_{1}$ and $m_{2}$ also increase with tensile strain. $m_{2}$ increases less rapidly than $m_{1}$ with increasing $\varepsilon$ and saturates around $0.25 \mu_{B}$ at $\varepsilon \sim+20 \%$. In contrast, $m_{1}$ seems to increase linearly with strain and has no saturation. The strain dependence of spin magnetization $m_{1}$ $\left(m_{2}\right)$ is the same as that of bond length $d_{1}\left(d_{2}\right)$ of $\mathrm{sp}^{2}(\mathrm{sp})$ bond, as shown in green in Fig. 3(a) and also as indicated by the linear dependence of magnetization $m_{1}\left(m_{2}\right)$ on bond length $d_{1}\left(d_{2}\right)$ in black dots in Fig. 3(b), suggesting contribution of strain-enhanced electron localization to the spin magnetization.

The localization of electrons with increasing tensile strain can be seen from the hopping integral values, too. Hopping integrals are parameters which monotonically decrease with increasing bond-length [14]. Figure 3(a) shows the bond-lengths $d_{1}$ and $d_{2}$ as a function of strain. Here $d_{1}$ and $d_{2}$ are defined in Fig. 1(a) as the bond-length of $\mathrm{sp}^{2}$ and sp bonds, respectively. $d_{1}$ increases with strain more abruptly than $d_{2}$, indicating the hopping integral $t_{1}$ of the $\mathrm{sp}^{2}$ bond drops more substantially than $\mathrm{t}_{2}$ of the $\mathrm{sp}$ bond. $t_{1}$ should be similar to the value in graphene due to a similar $\pi_{z}$ electron hopping on top of $\mathrm{sp}^{2}$ hybridization. $t_{2}$ has contributions from both $\pi_{x}$ and $\pi_{y}$ hopping channels on top of sp hybridization and is expected to be larger than $t_{1}$. The effective hopping integral $\tilde{t}$ from one hexagonal corner site to another is therefore expected to smaller than $t_{1}$ and decreases with increasing tensile strain faster than $t_{1}$. The calculated hopping integral confirms this trend, as shown in Fig. 3(a). The value of effective hopping integral $\tilde{t}$ is extracted from two ways, one of which $\left(\tilde{t}_{\alpha-\text { graphyne }}\right.$ in Fig. $\left.3(\mathrm{a})\right)$ is from the band gap $\mathrm{E}_{g}^{M}\left(\mathrm{E}_{g}^{M}=2 \tilde{t}\right)$ at the $\mathrm{M}$ point (the zone edge center point of hexagonal Brillouin zone), the other $\left(\tilde{t}_{\alpha-\text { graphyne }}^{b}\right.$ in Fig. 3(a)) based on $\tilde{t}\left(\sim-\mathrm{t}_{1}^{2} / \mathrm{t}_{2}\right)[10]$ and $\mathrm{t}_{i} \sim-0.63 \frac{\hbar^{2}}{m d_{i}^{2}}[14]$. From Fig. 3(a), $\tilde{t}_{\mathrm{s}}$ of $\alpha$-graphyne extracted from two ways are slightly different, but are both much less than that of graphene.

In order to understand the phenomena generally, we consider the Hubbard model (Eq. 1). The Hubbard model has been widely used to understand the strain-induced semimetal-antiferromagnetic semiconductor transition in graphene $[2,5,6,10]$. The electronelectron on-site Coulomb interaction U competes with electronic hopping integral t. Monte-Carlo calculations [1] based on the Hubbard model have predicted a critical value of $\mathrm{U} / \mathrm{t}$ in graphene $(\sim 4.3)$, over which energy gain due to electronic hopping can not counteract energy cost due to the Coulomb repulsion and transi-

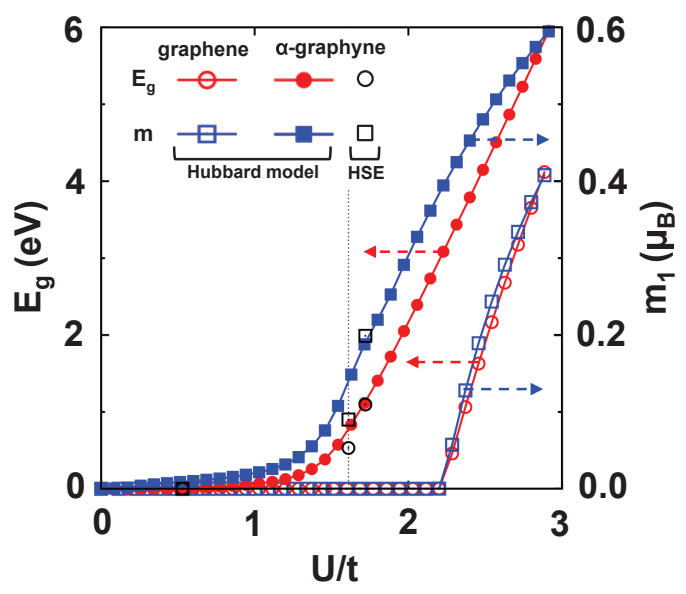

FIG. 4. (Color online) Energy band gap and magnetization $\mathrm{m}_{1}$ as a function of $\mathrm{U} / \mathrm{t}$ in $\alpha$-graphyne from Hubbard Model calculations. Graphene result from Hubbard Model calculations (blue open square and red open circles) and $\alpha$-graphyne from hybrid functional (HSE) calculations (black open square and circles) are also given for a comparison. A vertical dotted line at $\mathrm{U} / \mathrm{t}=1.61$ is to show the HSE result of $\alpha$-graphyne at $\varepsilon=0$. 
(a)

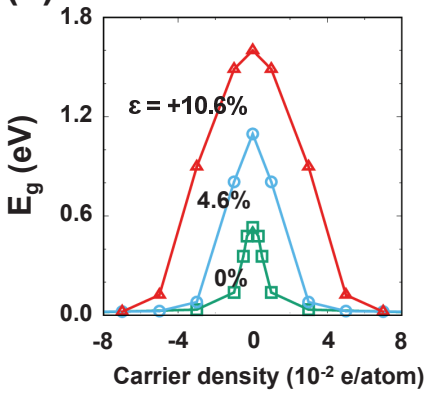

(b)

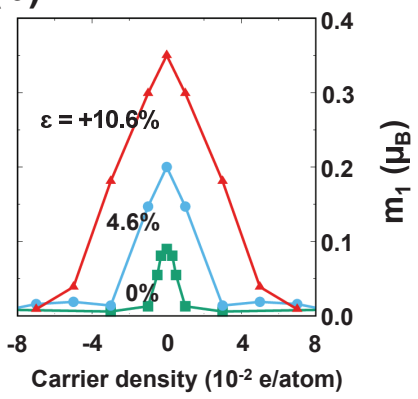

FIG. 5. (Color online) Carrier doping effect in $\alpha$-graphyne. (a) The band gap and (b) spin moment $m_{1}$ as a function of carrier density for tensile strain $\varepsilon$ from $0 \%$ to $10.6 \%$.

tion occurs. Within the Hartree-Fock mean-field approximation, we solved the Hubbard model of $\alpha$-graphyne and also of graphene for comparison. Figure 4 shows band gap $\mathrm{E}_{g}$ and magnetization $m_{1}$ as a function of $\mathrm{U} / \mathrm{t}$ for both $\alpha$-graphyne and graphene. We obtained the same critical value $(\mathrm{U} / \mathrm{t})_{c}$ in graphene as in literature using the same method $[25,26]$. It is reasonable that $(\mathrm{U} / \mathrm{t})_{c}(\sim 2.2)$ for graphene from Hatree-Fock mean field lies below the value $\left((\mathrm{U} / \mathrm{t})_{c} \sim 4.3\right)$ from Monte-Carlo calculations $[2,5,10]$. For comparison, $\alpha$-graphyne shows a similar trend of both band gap and magnetization with $\mathrm{U} / \mathrm{t}$ as graphene, but $\alpha$-graphyne has a much reduced and ill-defined critical value $\left((\mathrm{U} / \mathrm{t})_{c} \sim 1.5\right.$ from a linear extrapolation of the band gap at large $\mathrm{U} / \mathrm{t}$ ), suggesting an onset of transition to the AF state at much smaller strain cost than in graphene. By fitting the band structures of Hubbard model to the HSE band structures near the Fermi level in Fig. 2(c), we obtained the $\mathrm{U} / \mathrm{t}$ values of $0.53,1.61,1.72$ at a strain of $-3 \%, 0 \%$, $+4.6 \%$, respectively and showed the three data points in black open square and circles in Fig. 4. In graphene with high hopping integral, a sizable tensile strain $(\sim$ $+8 \%$ ) is predicted to be necessary for $\mathrm{U} / \mathrm{t}$ to approach the critical value $(\sim 2.2)[2,5,10]$, whereas compared with graphene, in $\alpha$-graphyne electronic hopping integral is much smaller, getting $\mathrm{U} / \tilde{t}$ in $\alpha$-graphyne at zero strain (A vertical dotted line in Fig. 4) already over the critical value and therefore the spontaneous antiferromagnetic semiconducting electronic ground state occurs. Further, when $\mathrm{U} / \tilde{t}$ increases with tensile strain, it stabilizes the AF state. The strain-enhanced stability of the AF ground state in $\alpha$-graphyne is advantageous for experimentalists to test this emergent phenomenon at finite temperature.

The strain-enhanced stability of the spontaneous AF insulator phase is also reflected in a competition between tensile strain and carrier doping. Carrier by either filling conduction band or emptying valence band tends to close the band gap and reduce spin magnetic moment, as shown in Fig. 5(a,b), since the energy gain by opening the energy gap is not expected. At zero strain, both band gap $\mathrm{E}_{g}$ and spin moment $m_{1}$ decrease with increasing (electron or hole) carrier density and approach zero at 0.03 electrons/atom (or a threshold carrier density at equivalently $1.7 \times 10^{14}$ electrons $\left./ \mathrm{cm}^{2}\right)$. This threshold carrier density is quite large compared with the value of graphene [3], indicating a good stability of antiferromagnetic state against the doping effect. The threshold value for $\mathrm{E}_{g}$ and $m_{1}$ increases with tensile strain as shown in Fig. 5. Moreover, the way of $\mathrm{E}_{g}$ and $m_{1}$ approaching zero is different from that of graphene. Lee et al. [3] found a bell-shaped decrease upon deviation from the band gap maximum in graphene, whereas in $\alpha$-graphyne we found a hat-shaped decrease of both $\mathrm{E}_{g}$ and $m_{1}$ from their maxima, in a much slower pace to get to zero. This hat-shaped behavior of both $\mathrm{E}_{g}$ and $m_{1}$ with carrier density in Fig. 5 is the same as that with $\mathrm{U} / \mathrm{t}$ in Fig. 4, suggesting that the phase transition from AF semiconductor to semi-metal in $\alpha$-graphyne is second-order.

In summary, we show a spontaneous AF semiconducting state in $\alpha$-graphyne by using hybrid functional calculations. Strain can increase the stability, band gap size at the $\mathrm{K}$ point and magnetic spin moments at each atomic site. The electronic hopping integral $\tilde{t}$ in $\alpha$-graphyne is found to be much smaller than that in graphene, which is essential to the understanding of such unusual spontaneous AF spin ordering. $\tilde{t}$ goes down with tensile strain, making AF state more robust and easier to test in experiment at non-zero temperature. The spontaneous AF insulator is quite robust against carrier doping effect, generating a hat-shaped decrease of both $\mathrm{E}_{g}$ and spin moment in $\alpha$-graphyne other than a bell-shaped decrease found in graphene. This study can be investigated by experiments in which strain and doping can be controlled systematically.

This project is supported by the National Key R\&D Program of China (No.2017YFA0206301) and the Major Program of Aerospace Advanced Manufacturing Technology Research Foundation NSFC and CASC, China (No. U1537204). R.S. acknowledges JSPS KAKENH Grant number JP25107005 and JP15K21722. H.G. acknowledges NSFC Grant No. 51702146, College Students' innovation and entrepreneurship projects (No. 201710148000072) and Liaoning Province Doctor Startup Fund (No. 201601325). B.J.D. and T.Y. acknowledge China Scholarship Council for financial support.

* yangteng@imr.ac.cn

[1] Z. Y. Meng, T. C. Lang, S. Wessel, F. F. Assaad, and A. Muramatsu, "Quantum spin liquid emerging in twodimensional correlated dirac fermions," Nature 464, 847851 (2010).

[2] T. O. Wehling, E. Şaşıŏlu, C. Friedrich, A. I. Lichtenstein, M. I. Katsnelson, and S. Blügel, "Strength of effective coulomb interactions in graphene and graphite," 
Phys. Rev. Lett. 106, 236805 (2011).

[3] Sung-Hoon Lee, Sungjin Kim, and Kinam Kim, "Semimetal-antiferromagnetic insulator transition in graphene induced by biaxial strain," Phys. Rev. B 86, 155436 (2012).

[4] Valeri N. Kotov, Bruno Uchoa, Vitor M. Pereira, F. Guinea, and A. H. Castro Neto, "Electron-electron interactions in graphene: Current status and perspectives," Rev. Mod. Phys. 84, 1067-1125 (2012).

[5] Ho-Kin Tang, E. Laksono, J. N. B. Rodrigues, P. Sengupta, F. F. Assaad, and S. Adam, "Interaction-driven metal-insulator transition in strained graphene," Phys. Rev. Lett. 115, 186602 (2015).

[6] Bitan Roy, Fakher F. Assaad, and Igor F. Herbut, "Zero modes and global antiferromagnetism in strained graphene," Phys. Rev. X 4, 021042 (2014).

[7] R. H. Baughman, H. Eckhardt, and M. Kertesz, "Structure-property predictions for new planar forms of carbon: Layered phases containing sp2 and sp atoms," The Journal of Chemical Physics 87, 6687-6699 (1987).

[8] Daniel Malko, Christian Neiss, Francesc Viñes, and Andreas Görling, "Competition for graphene: Graphynes with direction-dependent dirac cones," Phys. Rev. Lett. 108, 086804 (2012).

[9] Daniel Malko, Christian Neiss, and Andreas Görling, "Two-dimensional materials with dirac cones: Graphynes containing heteroatoms," Phys. Rev. B 86, 045443 (2012).

[10] Bog G. Kim and Hyoung Joon Choi, "Graphyne: Hexagonal network of carbon with versatile dirac cones," Phys. Rev. B 86, 115435 (2012).

[11] S. Das Sarma, Shaffique Adam, E. H. Hwang, and Enrico Rossi, "Electronic transport in two-dimensional graphene," Rev. Mod. Phys. 83, 407-470 (2011).

[12] W. P. Su, J. R. Schrieffer, and A. J. Heeger, "Solitons in polyacetylene," Phys. Rev. Lett. 42, 1698-1701 (1979).

[13] Riichiro Saito and Hiroshi Kamimura, "Vibronic states of polyacetylene, (ch)x," Journal of the Physical Society of Japan 52, 407-416 (1983), https://doi.org/10.1143/JPSJ.52.407.

[14] Walter A. Harrison, Elementary Electronic Structure, Re- vised Edition (World Scientific Publishing Co. Pte. Ltd., Singapore, 2004).

[15] P.W. Anderson, "Theory of magnetic exchange interactions:exchange in insulators and semiconductors," Solid State Physics 14, 99-214 (1963).

[16] Jochen Heyd, Gustavo E. Scuseria, and Matthias Ernzerhof, "Hybrid functionals based on a screened coulomb potential," The Journal of Chemical Physics 118, 82078215 (2003).

[17] G. Kresse and J. Furthmüller, "Efficient iterative schemes for $a b$ initio total-energy calculations using a plane-wave basis set," Phys. Rev. B 54, 11169-11186 (1996).

[18] G. Kresse and D. Joubert, "From ultrasoft pseudopotentials to the projector augmented-wave method," Phys. Rev. B 59, 1758-1775 (1999).

[19] John P. Perdew, Kieron Burke, and Matthias Ernzerhof, "Generalized gradient approximation made simple," Phys. Rev. Lett. 77, 3865-3868 (1996).

[20] Hendrik J. Monkhorst and James D. Pack, "Special points for brillouin-zone integrations," Phys. Rev. B 13, 5188-5192 (1976).

[21] M. R. Hestenes and E. Stiefel, "Methods of conjugate gradients for solving linear systems," J. Res. Natl. Bur. Stand. 49, 409-436 (1952).

[22] Young-Woo Son, Marvin L. Cohen, and Steven G. Louie, "Half-metallic graphene nanoribbons," Nature 444, 347349 (2006).

[23] Young-Woo Son, Marvin L. Cohen, and Steven G. Louie, "Energy gaps in graphene nanoribbons," Phys. Rev. Lett. 97, 216803 (2006).

[24] Gabor Zsolt Magda, Xiaozhan Jin, Imre Hagymasi, Peter Vancso, Zoltan Osvath, Peter Nemes-Incze, Chanyong Hwang, Laszlo P. Biro, and Levente Tapaszto, "Roomtemperature magnetic order on zigzag edges of narrow graphene nanoribbons," Nature 514, 608-611 (2014).

[25] S. Sorella and E. Tosatti, "Semi-metal-insulator transition of the hubbard model in the honeycomb lattice," EPL (Europhysics Letters) 19, 699 (1992).

[26] L. M. Martelo, M. Dzierzawa, L. Siffert, and D. Baeriswyl, "Mott-hubbard transition and antiferromagnetism on the honeycomb lattice," Zeitschrift für Physik B Condensed Matter 103, 335-338 (1996). 